\title{
Fuzzy Knowledge Representation for Linguistic Description of Time Series
}

\author{
A. Bugarín ${ }^{1}$ N. Marín ${ }^{2}$ D. Sánchez ${ }^{2,3}$ G. Trivino ${ }^{3}$ \\ ${ }^{1}$ Research Center on Information Technologies (CiTIUS), University of Santiago de Compostela. \\ ${ }^{2}$ Department of Computer Science and AI. University of Granada. \\ ${ }^{3}$ European Centre for Soft Computing.
}

\begin{abstract}
The linguistic description of data intends to provide texts that convey the most important information contained in the data. One of the main tasks to be carried out in order to build a linguistic description is the extraction and representation of the knowledge to be transmitted. To perform this task, adequate mechanisms for knowledge representation are needed. In this paper we focus on time series data and analyze three knowledge representation languages that arise in the field of Fuzzy Sets Theory, particularly Computing with Words and Perceptions: the use of Protoforms, the Granular Linguistic Model of a Phenomenon and, specially, the use of Fuzzy Temporal Knowledge Representation Models.
\end{abstract}

Keywords: Linguistic Description of Data, Time Series, Knowledge Representation, Protoforms, Granular Linguistic Model of a Phenomenom, Fuzzy Temporal Knowledge representation models, Fuzzy Temporal Rules.

\section{Introduction}

The development of systems for human-computer interaction based on natural language has played a relevant role in ICT research and is growing in importance today, due in part to the widespread use of mobile devices along with the many possibilities offered by technology to interact with these devices.

Within this context, the field of linguistic description of data (LDD) aims to provide in text format the most relevant information in data to interested users. This area of research is increasingly important as a bridge between the large amounts of data acquired by computer systems and the user interested in valid, valuable and useful knowledge contained in such data. Solutions within this area come more and more through the use of integrated approaches where tools and techniques of disciplines like Natural Language Generation and processing, Soft Computing, and Intelligent Data Analysis and Data Mining, converge and complement each other.

In this area of research it is widely recognized the contribution that uncertainty representation techniques, particularly those related to the Fuzzy Set Theory and its extensions, can provide. Thanks to
Fuzzy Set Theory, models can be designed which are more understandable, less complex and more robust, being this theory especially useful for the representation of vague patterns and the modeling and processing of various forms of uncertain and incomplete information [1]. In the community of Natural Language Generation (NLG), the role that this theory can play to cover the semantic gap between data and linguistic expressions is also recognized, for instance in the management of different types of uncertainty which are inherent to the linguistic expression of knowledge about real world data $[2,3,4]$. This is in line with the known suitability and the high potential of fuzzy sets to represent the semantics of natural language expressions [5].

From a general point of view, in order to provide a linguistic description of the data, two main tasks must be performed, which are: to extract from the data the knowledge to be transmitted, and to generate an appropriate linguistic expression of the knowledge for the target user. The first task, by its nature, can be considered a Knowledge Data Discovery (KDD) process, which aims to provide a collection of messages that computationally represent the knowledge that is in the data. This requires defining a knowledge representation formalism that allows to represent the semantics of the messages. The expressive power of this formalism defines the space of possible messages to be considered as base for the construction of the final description.

Frequently, data is presented in the form of time series. In a broad sense, a time series can be considered a data stream produced by a given phenomenon ordered in time. In this work, we study three relevant approximations to the problem of knowledge representation in linguistic description of time series data that arise in the field of Fuzzy Set Theory, namely: the use of protoforms, the Granular Linguistic Model of a Phenomenon, and Fuzzy Temporal Models.

In the next section, we analyze the three mentioned approaches to the problem of knowledge representation; finally, in Section 4, we present our conclusions and point out some guidelines for future work in the area. 


\section{Some Fuzzy Knowledge Representation Elements for Linguistic Description of Data}

In the following sections we describe different knowledge representation languages that have been employed to a larger or lesser extent in the literature. It is important to remark that these languages are not three alternatives to the same problem, but they cover different aspects of knowledge representation. In fact, they have been frequently combined for developing systems for linguistic description of data.

\subsection{Protoforms}

Protoforms (prototypical forms) arise within the Computing with Words and Perceptions paradigm proposed by L.A. Zadeh, and the closely related Generalized Theory of Uncertainty (GTU) [6]. A protoform is defined as an abstracted model which instances represent knowledge about data [7]. They are particularly useful in formalizing human consistent reasoning and for providing deduction capabilities, specially in natural language-based knowledge discovery tools [8].

Some of the most widely employed protoforms are:

- The protoform $X$ is A, defining a possibilistic constraint. Examples of instances are "Temperature is High", "Wind Speed is $33 \mathrm{mph}$ ", and "Stock price variation is highly increasing".

- The protoform $Q D$ are $A$, where $Q$ is a linguistic quantifier, and $D$ and $A$ are fuzzy subsets of the same universe of reference defined by fuzzy predicates. This is a particular case of fuzzy quantified sentence $[9,6]$. Examples of instances are "Most days are foggy" and "Usually between 12 and $15 \mathrm{~h}$. the price is constant". Kacprzyk and Zadrozny [8] point out that protoforms can be seen as a general form of a linguistic data summary, a term coined by Yager for a specific kind of linguistic description based on quantified guided aggregation of data [10], and it is very frequently used as a synonym for linguistic description even in the NLG community, see for instance [11].

Protoforms are useful for dealing with different kinds of uncertainty inherent to the linguistic expression of knowledge about real world data. For instance, the protoform $X$ is $A$ represents the (partial) knowledge we have about the actual value $a$ of the variable $X$ (we only know $a$ is in $A$, being the membership function $A(x)$ a measure of the possibility that a certain value $x$ is the actual value of $X)$. However, in the setting of linguistic description of data, in which the values of the time series are usually known, the expression $X$ is $A$ is generally given a different use, particularly as a way to connect data values to linguistic terms in a kind of change of granularity.
Knowledge representation in linguistic description of time series is employed in order to represent the semantics of messages through the semantics of protoforms and those of their instance's components, which are the basic elements of any formalism. For example, the semantics of the message represented by the instance "Usually between 12 and $15 \mathrm{~h}$. the price is constant" is given by the semantics of:

- the protoform $Q D$ are $A$ (the fraction of objects in $D$ that are in $A$ is $Q$ )

- the component between 12 and $15 \mathrm{~h}$. (represented by a crisp set),

- the component constant (which, when measuring the variation of a time series by means of the angle with the horizontal axis of a line adjusted to the series, can be represented by a fuzzy subset of angles around zero),

- the component Usually (a fuzzy relative quantifier represented by a non-decreasing fuzzy subset of $[0,1]$ )

as well as a collection $\Omega$ of time segments (taken from a single series or from different series). Then, $D$ is the (in this case crisp) set of intervals with boundaries 12 and $15 \mathrm{~h}$. in $\Omega$, and $A$ is the fuzzy subset of $\Omega$ satisfying that the variation of the time series is constant in the sense explained before.

A very important aspect of protoforms is the assessment of protoform instances by measuring the degree to which the knowledge expressed by the instance is an accurate description of the data. The assessment of the accuracy of instances of $X$ is $A$ is very simple, and given by the membership of the actual value of $X$ to the fuzzy set $A$, having been employed by many authors; for instance, in [12] $A$ is a categorical value or a crisp interval of numerical values, ordered in time, whilst in $[13] X$ represents a trend or the sign of a trend, and $A$ is a label obtained by combining basic linguistic labels and linguistic hedges.

In the case of quantified sentences, there are many different approaches that have been employed in practical LDD applications, like Zadeh's approach based on compatibility of a quantifier with the sigma-count cardinality [14], the Sugeno integral [15], the Choquet integral [16], Yager's method based on OWA [17], method GD in [18, 19], and methods based on generalized quantification in $[20,21,22,23,24]$. A review of techniques for the evaluation of quantified sentences including these and other methods can be found in [25].

\subsection{Granular Linguistic Model of a Phenomenon}

The Granular Linguistic Model of a Phenomenom (GLMP) is a hierarchically organized knowledge representation model for linguistic description. A formal description of the model is presented in [26]. Figure 1 shows a simple example of GLMP. The 
nodes of the hierarchy represent perceptions, each one being usually expressed in practice by means of an associated protoform. There is no restriction as to which protoforms can be employed; in fact, the (many) applications in which this model has been applied use a wide variety of protoforms, even in the same application.

A directed link between two perceptions in the hierarchy indicates that the first perception explains the second, meaning that protoform instances of a certain perception are calculated by inference on the basis of instances of the perceptions which explain it, using generally fuzzy rules and Mamdani-based inference, but allowing for other kinds of inference. Perceptions which are explained by the input data are called first order perceptions $\left(\hat{P}_{1}^{1}, \hat{P}_{2}^{1}\right.$ and $\hat{P}_{3}^{1}$ in Figure 1), and are usually associated to low-level protoforms like $X$ is $A$ or quantified sentences. Perceptions explained by other perceptions are called second order perceptions $\left(\hat{P}_{4}^{2}\right.$ and $\hat{P}_{5}^{2}$ in Figure 1$)$. The model allows the representation of uncertainty about the perceptions (represented by $W_{i}$ in Figure 1 ), as well as associating specific linguistic expression mechanisms to each perception.

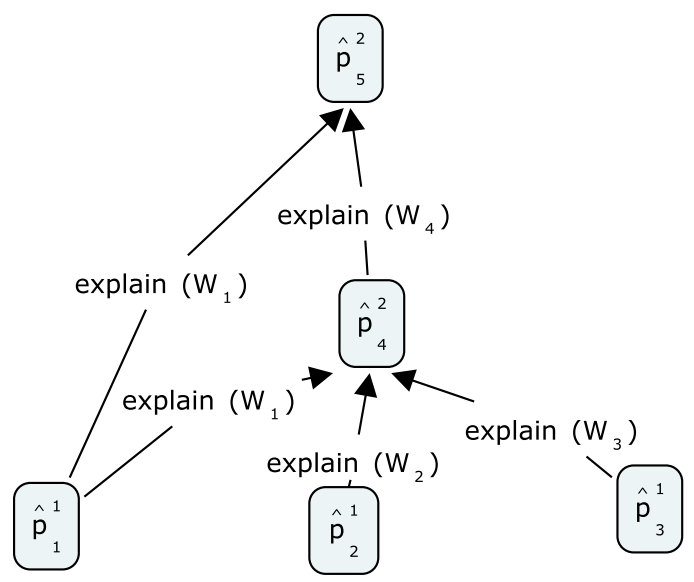

Figure 1: Simple example of GLMP.

This model has been employed in many applications. For instance, in the linguistic description of the human gait quality $[27,28]$, the description of rehabilitation exercises [29], the linguistic description of energy consumption data of households [30], reporting in driving simulation environments [31], description of perceptions while driving [32], and the linguistic description of traffic $[33,34]$, among others.

Figure 2 shows a GLMP used to describe the physical style of life of a person. The first order perceptions are obtained from the accelerations provided by using a smartphone carried by the subject. $\rho$ is the acceleration modulus and $\sigma$ is the typical deviation of this temporal signal.

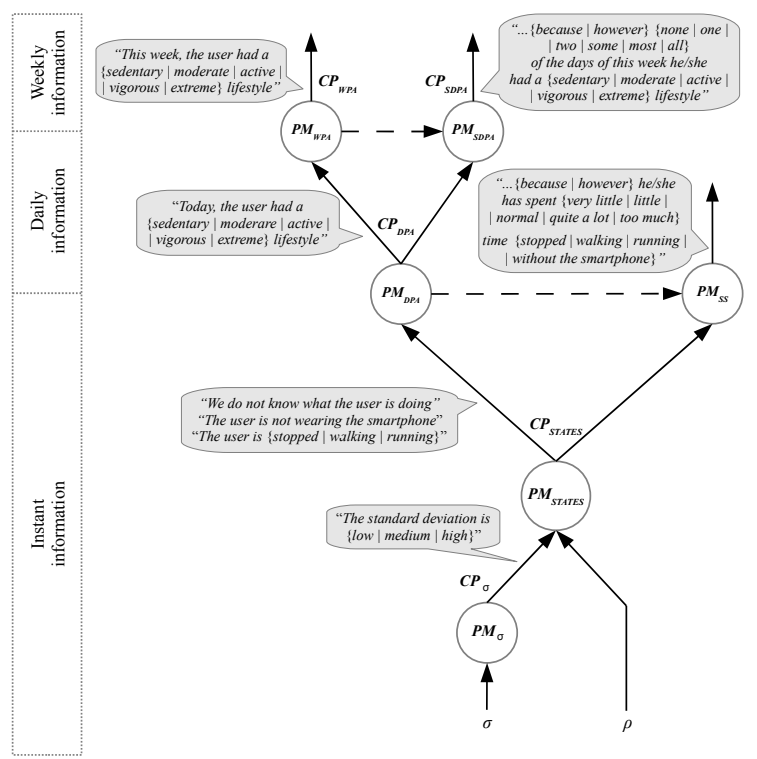

Figure 2: GLMP used to describe the physical style of life of a person

\subsection{Fuzzy Temporal Models}

It is well-known that linguistic reporting from time series data in non-trivial fields of application demands the use of expressions where time explicitly appears, either as a temporal reference or as a framework for the occurrence of events. Some examples of descriptions, taken from the process monitoring and control areas are: The distance to the wall has kept low in part of the last measurements or The temperature rose considerably a little before that the pressure became high. Other expressions involving different types of operators or describing complex relations among values of variables or relevant events are also frequent in these and related areas (e.g. for a minute the mean value of the temperature in boiler 1 was bigger than the mean value of the temperature in boiler 2). These expressions pose knowledge representation needs that do not fit neither within the classical non-temporal fuzzy protoforms $X$ is $A$ or the quantified ones $Q$ Bs are As mentioned in Section 2.1. Modelling complex expressions in real and demanding application realms requires to use adequate models that take into account an explicit fuzzy representation of time and consider that fuzziness can appear not only in the temporal references, but also in the sets of values and the operators involved in the descriptions.

Although many models have been proposed in the literature for fuzzy temporal knowledge representation and reasoning [35], [36], [37], [38], [39], [40], [41], [47] to explicitly include time as another decision variable in fuzzy propositions and rules, they have received little attention, in general, from re- 
searchers in the LDD area. Nevertheless, many of these models are valuable sources of inspiration for endowing LDD models with the syntactic expresiveness and linguistic richness many applications and users demand. If we aim that LDD may face truly complex real problems, the underlying models should be endowed with knowledge representation elements that may allow the descriptions produced to be close to the language used by human users (that should be always in the center of interest of LDD).

Our starting point of view it that many of the approaches described in the LDD literature do not produce, in general, texts that are adequate for direct human consumption by non-specialized users. Linguistic summaries in these cases are usually made up of limited expressions that involve simple type I or type II quantified propositions $(Q X s$ are $A$ or $Q B s$ are $A s$ ) where $Q$ is a basic absolute/relative quantifier. Therefore, these models lose most of the semantic richness that many models described in the fuzzy knowledge representation and reasoning literature are endowed with. Since LDD is finally addressed to human consumption it should be a fundamental requisite that they are based on sound knowledge representation models that are truly able to fit the users semantic needs and not on the contrary. It seems that most of these LDD models have been described exclusively from a computational point of view, thus forgetting to look for inspiration on other fields (such as linguistics or logics, but also in process supervision and control, dynamic or complex systems or even signal processing) where similar problems and representation needs have been successfully dealt with for long time.

In many of these areas systems are usually described as the evolution of a sequence of pre-defined states. Transitions between states are modelled in this field by means of fuzzy knowledge bases, since they represent complex change situations where the context, involving temporal relationships among other states or variables, should be taken into consideration.

A model for representing dynamic behavior, called Fuzzy Finite State Machine (FFSM), has been proposed by Trivino et al. [32]. A FFSM is formally defined as a tuple:

$$
\left\{X, U, Y, f, g, X_{0}\right\}
$$

where:

- $X$ is the set of states $\left\{x_{1}, x_{2}, \ldots, x_{n_{x}}\right\}$. Every state represents the pattern of a repetitive situation. We say that, the system is in a specific state, when the current input variables and the previous state activations fulfill certain conditions. The activation of a state is a matter of degree, i.e. the FFSM could be partially in several states simultaneously. We will denote $\mathcal{X}_{i} \in[0,1]$ the degree of activation of a state,
Defining the states includes determining their temporal order, i.e., the sequence with which the system follows the different relevant states.

- $U$ is the input vector $\left\{u_{1}, u_{2}, \ldots, u_{n_{u}}\right\}$. $\mathrm{U}$ is a set of first-order perceptions where each variable $u_{i}$ takes its value in a domain defined with a set of linguistic labels $\left\{A_{i 1}, A_{i 2}, \ldots, A_{i n}\right\}$.

- $Y$ is the output vector $\left\{y_{1}, y_{2}, \ldots, y_{n_{y}}\right\}$. $Y$ represents a summary of the values taken by the inputs while staying in a specific state.

- $f$ is the state transition function $X[t+1]=$ $f(U[t], X[t])$. This function can be implemented using a set of fuzzy rules:

- Rules that constrain the signal amplitude. We distinguish between rules to stay in a state $x_{i}\left(R_{i i}\right)$ and rules to change from the state $x_{i}$ to the state $x_{j}\left(R_{i j}\right)$ :

$R_{i i}:$ IF $\mathcal{X}_{i}(t) \vee\left(u\right.$ is $\left.C_{i}\right)$ THEN $\mathcal{X}_{i}(t+1)$ $R_{i j}:$ IF $\mathcal{X}_{i}(t) \wedge\left(u\right.$ is $\left.C_{j}\right)$ THEN $\mathcal{X}_{j}(t+1)$ where $C_{i}$ and $C_{j}$ represent the conditions of amplitude for the state $x_{i}$ and $x_{j}$ respectively.

$\checkmark$ in $R_{i i}$ is used to introduce an inertia to change of state. This makes the FFSM more robust against spurious in the input. This OR is typically implemented using the Maximun operator. $\wedge$ in $R_{i j}$ is used to define more sharply the conditions to change. This AND is typically implemented using the Minimum operator.

- Rules that constrain the signal time span. Two additional linguistic labels are used for this purpose:

Time_to_stay ${ }_{i}$ : is the maximum time that the signal is expected to remain in state $x_{i}$.

Time_to_change $e_{i j}$ : is the minimum time that the signal is expected to remain in state $x_{i}$ before changing to state $x_{j}$. Therefore, adding the temporal conditions:

$$
\begin{aligned}
& R_{i i}: \quad \operatorname{IF} \mathcal{X}_{i}(t) \vee\left(u=C_{i}\right) \vee\left(d_{i}=\right. \\
& \left.T_{\text {stay }_{i}}\right) \text { THEN } \mathcal{X}_{i}(t+1) \\
& R_{i j}: \quad \operatorname{IF} \mathcal{X}_{i}(t) \wedge\left(u=C_{j}\right) \wedge\left(d_{i}=\right. \\
& \left.T_{\text {change }_{i j}}\right) \text { THEN } \mathcal{X}_{j}(t+1) \\
& \text { where } d_{i} \text { is the duration of the state } x_{i} .
\end{aligned}
$$

- $g$ is the output function $Y[t]=g(U[t], X[t])$. The output variables are obtained as a summary of the values of the inputs while the system remained in the considered state, e.g. using the average or the standard deviation (See an example in the next section).

- $X_{0}$ is the initial state.

Figure 3 shows the states diagram of a FFSM used to recognize different states of activity of a person. This FFSM is used to implement $P M_{S T A T E S}$ in the previous example where we present a GLMP that describes the physical style of life of a person 


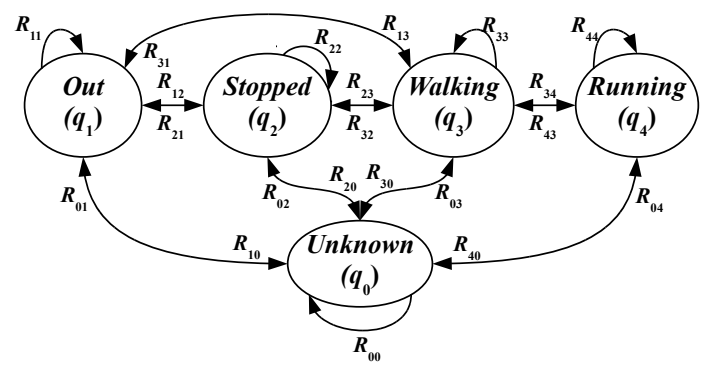

Figure 3: This FFSM is used to implement $P M_{S T A T E S}$ in the GLMP that describes the physical style of life of a person

\subsubsection{Fuzzy Representation of Time}

Although [40] firstly formalised the representation of fuzzy time by means of intervals associated with fuzzy sets, the most important work regarding the formalisation of fuzzy time was carried out in [41], where the basis for the representation of imprecision and uncertainty in temporal knowledge within the framework of the possibility theory were established. Taking time points as primitives, basic concepts are introduced, such as fuzzy instant and fuzzy temporal interval, shaping the possible temporal relations between these temporal entities through fuzzy relations. With some variations, these concepts and the way of representing them appear in the majority of the works related to fuzzy temporal knowledge representation. Other models [35] handle Allen's basic temporal relations [42] between temporal entities and are able to model expressions where temporal relations are established between the occurrence of different events, such as in temperature increased a few minutes after pressure decreased. In [38] a model is presented introducing a nonlinear perception of time, giving more precision, reliability and certainty to the observations and actions closer in time, by means of the fading of the fuzzy temporal sets. In [39] a temporal proposition, assumed to describe a situation valid for a given time period, is split into two components: a non-temporal component and a time-dependent component. In the medical domain, [43] introduced a generic language (Patient Case Report Language: PCRL) for patient case reports, based in general medical temporal concepts and focusing on the modelling of the concepts expressed in natural language. The problem of temporally relate time intervals is reduced to temporally relate time points (instants) since intervals are fully characterized by the (fuzzy) instants they begin and end at. The concept of quantification is used to express information like: all the episodes in the last 2 weeks when the headache lasted no more than 2 hours, and is extended to allow quantifier margins (at least, at most, precisely...), making the quantification less precise and more adapted to medical descriptions.
In general, there are some aspects related to the semantic richness of the temporal expressions used by a human expert that are not satisfactorily covered with these models. An example is the concept of persistence: when a condition on a variable must be evaluated over a fuzzy temporal interval, it may be required that the condition is fulfilled, to some degree, for all the time points in the interval, or just for any of them. Between both extremes there is a range of intermediate situations (in most of the previous hour) not yet fully considered by the previous models. In this regard, the proposal in [44], and more extensively [47], presents formal proposals for time quantification in a temporal expressions. In particular, the Fuzzy Temporal Propositions model in [47] also describes a temporal ontology and language than permits the explicit modelling of relative occurrences among events, fuzzy quantification and a number of other operators of interest. Many of the models for the elements in the ontology are compiled or taken from other temporal knowledge models previously mentioned [41, 42], but also from other areas (quantification models [9, 48], Zadeh's Extension Principle [50], Fuzzy Arithmetics, ...). All these elements may be seen as a catalogue that provides models that can be taken into consideration for the definition of language specifications in LDD models that explicitly manage temporal information.

\subsubsection{Some Fuzzy temporal elements for $L D D$ specification}

\section{Temporal primitives}

From the temporal model in $[41,45]$, the following basic temporal primitives should be considered:

- Fuzzy temporal instant, described through a possibility distribution whose support elements represent mutually exclusive candidates for an imprecise instant defined as a disjunctive set (e.g. around eight o'clock).

- Fuzzy temporal duration, represented by means of a possibility distribution on the set of the integers that represent time units (e.g. around six hours).

- Fuzzy temporal interval, which is a time period defined by two of the following three elements: an initial fuzzy instant, an ending fuzzy instant, and the interval duration. It is a possibility interval that represents the conjunctive fuzzy set of all the time points possibly comprised between the start and the end of the interval.

- Fuzzy temporal relations: all the basic temporal relations (both qualitative and quantitative [45]) can be considered (e.g. before, at the end of, when, ...). In particular, Allen's temporal interval relations [42], which have been extended in [46] to fuzzy intervals are well-known models. 


\section{Temporal constraints}

Temporal constraints can be fuzzy instants or intervals. Intervals can also act as a general temporal context [43] or temporal window for an event. Absolute temporal constraints related to a fixed temporal entity (at 20:00), or to the current time point (ten minutes ago), whereas relative temporal constraints depend on the occurrence time (imprecise) point of other event/s ( a little after an increase in pressure, between 30 minutes and 2 hours after the beginning of the irradiation). When the temporal constraint is an interval, the previously mentioned concept of persistence of an event within an interval naturally arises, giving way to the use of temporal quantification of propositions and, therefore, to fuzzy quantification models. The occurrence of an event may happen at all the time points in the temporal interval, at a single time point in the interval, or be required in a partial way. Fuzzy quantifiers allow to linguistically model these situations as: $X$ is $A$ in $Q$ of $T$, being $X$ a variable, $A$ a linguistic value, $Q$ a fuzzy quantifier and $T$ a temporal interval. In the example the temperature has been high most of the time between $18 \mathrm{~h}$ and $21 \mathrm{~h}$ ) we have $X=$ temperature, $A=$ high,$Q=$ most and $T=$ previous half an hour. As pointed out in previous sections, fuzzy quantification has been very widely dealt with in the literature from many research points of view and a number of models have been proposed and characterized in the fuzzy and linguistics literature $[48,49,25]$.

\section{Fuzzy specification operators}

A fuzzy specification operator selects a candidate among several values, according to fuzzy nontemporal or temporal criteria. Examples of this type of operators are terms like first, last, maximum, minimum, like in the maximum of pressure values in the last 30 minutes, the last value of high temperatures or the minimum of the velocity values in the proximity of an obstacle. In the crisp case, a specification operator simply selects the corresponding crisp value among the different candidates: in the first example, it would take the greatest of the pressure values in the last 30 minutes. But in the fuzzy case the value selected from the operator will not necessarily be a crisp and unique value, but a possibility distribution. In this case, Zadeh's extension principle $[9,50]$ provides a wellknown sistematic tool form building fuzzy models of operators starting from crisp definitions. Other approaches based on $\alpha$-cuts [49] or $\gamma$-cuts [48] have also been considered. Analysis of the properties of the extended models becomes here a crucial issue, since it has been shown for very well-known cases (e.g. fuzzy arithmetics, fuzzy quantifiers) that the extended models do not always keep intuitive properties that hold in the crisp models the come from.

\section{Fuzzy reduction operators}

Reduction operators area applied on data within on a temporal reference, to obtain a new single value that somehow numerically summarizes the original ones. Some examples are mean value or accumulated value. In many application areas these operators are modelled assuming a crisp definition. Nevertheless, when the operator is applied on fuzzy values (e.g. the mean of high temperatures), it is necessary again to extend crisp definitions to the fuzzy case by using any of the extension models previously indicated $[9$, $50]$.

\section{Conclusions}

Fuzzy Sets Theory offers a wide range of techniques that are potentially useful in the challenge of obtaining linguistic descriptions of time series data, among other topics, in relation to the problem of knowledge representation which the construction of the descriptions involves.

In this paper, we have shown that the representation of knowledge in such systems can be done through more or less structured collections of protoforms, in which, in many cases, quantification plays a fundamental role. Though the number of significant proposal in the literature is remarkable and increasing, there are many results in the field of fuzzy quantification (fuzzy cardinality and entropy, probability of fuzzy events and fuzzy probabilities, ...) which can play an important role in achieving more powerful systems.

Among the more structured representations using protoforms, the so called Granular Linguistic Model of a Phenomenom is a remarkable approach, where perceptions and inference mechanisms are mixed to build a knowledge representation of problems of some complexity.

Finally, we have pointed out that more attention should be paid to the treatment of time and temporal relations in knowledge representation models. Research initiatives that focus on the representation of imprecision and uncertainty in the temporal knowledge within the Theory of Possibility would improve the variety of tools for linguistic description of data sets. Though the inclusion of time as a principal variable in fuzzy propositions and rules has received insufficient attention in the literature, its solvent use should serve to address issues of complexity and bring the description closer to the language used by human users. Although many studies have emerged to complete initial works in the literature, there are still many open challenges regarding temporal primitives, constraints and other fuzzy operators.

\section{Acknowledgments}

This work has been supported in part by Junta de Andalucía, Spain, under grant P11-TIC-7460 and 
the Spanish Ministry of Economy and Competitiveness under grants TIN2011-29827-C02-01/02 and TIN2014-58227 "Descripción Lingüística de Información Visual mediante Técnicas de Minería de Datos y Computación Flexible".

\section{References}

[1] E. Hüllermeier, Fuzzy sets in machine learning and data mining, Applied Soft Computing 11 (2) (2011) 1493 - 1505.

[2] K. van Deemter, Utility and language generation: The case of vagueness, J. Philosophical Logic 38 (6) (2009) 607-632.

[3] K. van Deemter, Vagueness facilitates search, in: M. Aloni, H. Bastiaanse, T. de Jager, K. Schulz (Eds.), Amsterdam Colloquium on Logic, Language and Meaning, Vol. 6042 of Lecture Notes in Computer Science, Springer, 2009, pp. 173 182.

[4] F. Portet, A. Gatt, Towards a possibilitytheoretic approach to uncertainty in medical data interpretation for text generation, in: D. Riaño, A. ten Teije, S. Miksch, M. Peleg (Eds.), KR4HC, Vol. 5943 of Lecture Notes in Computer Science, Springer, 2009, pp. 155-168.

[5] L.A. Zadeh, From computing with numbers to computing with words - from manipulation of measurements to manipulation of perceptions, Appl. Math. Comput. Sci 307-324.

[6] L.A. Zadeh, Generalized theory of uncertainty (GTU) - principal concepts and ideas, Computational Statistics \& Data Analysis 51 (2006) $15-46$.

[7] L. A. Zadeh, A prototype-centered approach to adding deduction capability to search enginesthe concept of protoform, in: Fuzzy Information Processing Society, 2002. Proceedings. NAFIPS. 2002 Annual Meeting of the North American, 2002, pp. 523-525.

[8] J. Kacprzyk, S. Zadrozny, Linguistic database summaries and their protoforms: towards natural language based knowledge discovery tools, Inf. Sci. 173 (4) (2005) 281-304.

[9] L. A. Zadeh, A computational approach to fuzzy quantifiers in natural languages, Computing and Mathematics with Applications 9 (1) (1983) 149-184.

[10] R. R. Yager, On linguistic summaries of data, in: Knowledge Discovery in Databases, 1991, pp. 347-366.

[11] S. Sripada, E. Reiter, J. Hunter, J. Yu, Generating english summaries of time series data using the gricean maxims, in: Proc. 9th ACM SIGKDD Int. Conf. on Knowledge discovery and data mining (KDD), 2003, pp. 187-196.

[12] E. Reiter, S. Sripada, J. Hunter, J. Yu, I. Davy, Choosing words in computer-generated weather forecasts, Artif. Intell. 167 (1-2) (2005) 137-169.
[13] V. Novák, V. Pavliska, I. Perfilieva, M. Stepnicka, F-transform and fuzzy natural logic in time series analysis, in: J. Montero, G. Pasi, D. Ciucci (Eds.), EUSFLAT Conf., Atlantis Press, 2013.

[14] R. J. Almeida, M.-J. Lesot, B. BouchonMeunier, U. Kaymak, G. Moyse, Linguistic summaries of categorical time series for septic shock patient data, in: FUZZ-IEEE, IEEE, 2013, pp. $1-8$.

[15] J. Kacprzyk, A. Wilbik, S. Zadrozny, Linguistic summaries of time series via a quantifier based aggregation using the sugeno integral, in: FUZZ-IEEE, IEEE, 2006, pp. 713-719.

[16] J. Kacprzyk, A. Wilbik, S. Zadrozny, Linguistic summarization of time series by using the choquet integral, in: P. Melin, O. Castillo, L. T. Aguilar, J. Kacprzyk, W. Pedrycz (Eds.), IFSA (1), Vol. 4529 of Lecture Notes in Computer Science, Springer, 2007, pp. 284-294.

[17] J. Kacprzyk, A. Wilbik, S. Zadrozny, Linguistic summaries of time series via an owa operator based aggregation of partial trends, in: FUZZIEEE, IEEE, 2007, pp. 1-6.

[18] R. Castillo-Ortega, N. Marín, D. Sánchez, Linguistic query answering on data cubes with time dimension, International Journal of Intelligent Systems 26 (10) (2011) 1002-1021.

[19] R. Castillo-Ortega, N. Marín, D. Sánchez, A fuzzy approach to the linguistic summarization of time series, Journal of Multiple-Valued Logic and Soft Computing 17 (2-3) (2011) 157-182.

[20] F. Díaz-Hermida, , A. Bugarín, S. Barro, Definition and classification of semi-fuzzy quantifiers for the evaluation of fuzzy quantified sentences, International Journal of Approximate Reasoning 34 (1) (2003) $49-88$.

[21] F. Díaz-Hermida, P. Cariñena, A. Bugarín, Efficient evaluation of similarity quantified expressions in the temporal domain, in: J. Lawry, E. Miranda, A. Bugarín, S. Li, M. A. Gil, P. Grzegorzewski, O. Hryniewicz (Eds.), SMPS, Vol. 37 of Advances in Soft Computing, Springer, 2006, pp. 191-198.

[22] F. Díaz-Hermida, A. Ramos-Soto, A. Bugarín, On the role of fuzzy quantified statements in linguistic summarization of data, in: S. Ventura, A. Abraham, K. J. Cios, C. Romero, F. Marcelloni, J. M. Benítez, E. L. G. Galindo (Eds.), ISDA, IEEE, 2011, pp. 166-171.

[23] F. Díaz-Hermida, A. Bugarín, Semi-fuzzy quantifiers as a tool for building linguistic summaries of data patterns, in: FOCI, IEEE, 2011, pp. $45-52$.

[24] A. Ramos-Soto, A. Bugarín, S. Barro, J. Taboada, Automatic generation of textual short-term weather forecasts on real prediction data, in: H. L. Larsen, M. J. Martín-Bautista, M. A. Vila, T. Andreasen, H. Christiansen (Eds.), FQAS 2013, Granada, Spain, Proceed- 
ings, Vol. 8132 of Lecture Notes in Computer Science, Springer, pp. 269-280, 2013.

[25] M. Delgado, M. D. Ruiz, D. Sánchez, M. A. Vila, Fuzzy quantification: a state of the art, Fuzzy Sets and Systems 242 (2014) 1 - 30.

[26] G. Triviño, M. Sugeno, Towards linguistic descriptions of phenomena, Int. J. Approx. Reasoning 54 (1) (2013) 22-34.

[27] A. Alvarez-Alvarez, G. Triviño, Linguistic description of the human gait quality, Eng. Appl. of AI 26 (1) (2013) 13-23.

[28] D. Sanchez-Valdes, G. Triviño, Computational perceptions of uninterpretable data. A case study on the linguistic modeling of human gait as a quasi-periodic phenomenon, Fuzzy Sets and Systems 253 (2014) 101-121.

[29] L. González-Villanueva, A. Alvarez-Alvarez, L. Ascari, G. Triviño, A tool for linguistic assessment of rehabilitation exercises, Appl. Soft Comput. 14 (2014) 120-131.

[30] A. van der Heide, G. Triviño, Automatically generated linguistic summaries of energy consumption data, in: Proceedings ISDA 2009, pp. 553-559, IEEE 2009.

[31] L. Eciolaza, M. Pereira-Fariña, G. Triviño, Automatic linguistic reporting in driving simulation environments, Appl. Soft Comput. 13 (9) (2013) 3956-3967.

[32] G. Triviño, A. van der Heide, An experiment on the description of sequences of fuzzy perceptions, in: F. Xhafa, F. Herrera, A. Abraham, M. Köppen, J. M. Benítez (Eds.), HIS, IEEE Computer Society, 2008, pp. 228-233.

[33] G. Triviño, A. Sanchez, A. S. Montemayor, J. J. Pantrigo, R. Cabido, E. G. Pardo, Linguistic description of traffic in a roundabout, in: Proceedings FUZZ-IEEE 2010, pp. 1-8.

[34] A. Alvarez-Alvarez, D. Sanchez-Valdes, G. Triviño, Á. Sánchez, P. D. Suárez, Automatic linguistic report of traffic evolution in roads, Expert Syst. Appl. 39 (12) (2012) 11293-11302.

[35] D. Qian, Representation and use of imprecise temporal knowledge in dynamic systems, Fuzzy Sets and Systems, 50:59-77, Elsevier, 1992.

[36] J. Virant, N. Zimic, Attention to time in fuzzy logic, Fuzzy Sets and Systems, 82:39-49, Elsevier, 1996.

[37] H. Maeda, S. Asaoka, S. Murakami, Dynamical fuzzy reasoning and its application to system modeling, Fuzzy Sets and Systems, 80:101-109, Elsevier, 1996.

[38] B. Carse, T. Fogarty, A. Munro, Artificial evolution of fuzzy rule bases which represent time: a temporal fuzzy classifier system, International Journal of Intelligent Systems, 13:905-927, Wiley, 1998.

[39] S. Raha and K. Ray, Approximate reasoning with time, Fuzzy Sets and Systems, 107:59-79, Elsevier, 1999.
[40] M. Vitek, Fuzzy information and fuzzy time. In proceedings of the IFAC Symposium on Fuzzy Information, Knowledge Representation and Decision Analysis, pages 159-162, 1983.

[41] D. Dubois, H. Prade, Processing fuzzy temporal knowledge, IEEE Transactions on Systems, Man and Cybernetics, 19:729-744, IEEE Press, 1989.

[42] J. Allen, Towards a general theory of action and time, Artificial Intelligence, 23:123-154, Elsevier, 1984.

[43] A. van der Maas, A. ter Hofstede, P. de Vries Robbé, Formal description of temporal knowledge in case reports, Artificial Intelligence in Medicine, 16:251-282, Elsevier, 1999.

[44] T. Kiseliova, H. Wagner, A generalized time quantifier approach to approximate reasoning, Fuzzy Sets and Systems, 145:213-228, Elsevier, 2004.

[45] S. Barro, R. Marín, J. Mira, A. Patón, A model and a language for the fuzzy representation and handling of time, Fuzzy Sets and Systems, 61:153-175, Elsevier, 1994.

[46] S. Schockaert, M. D. Cock, E. Kerre, Fuzzifying Allen's temporal interval relations, IEEE Transactions on Fuzzy Systems, 16:517-533, IEEE Press, 2008.

[47] P. Cariñena, A. Bugarín, M. Mucientes, F. Díaz-Hermida, S. Barro, Fuzzy Temporal Rules: A Rule-based Approach for Fuzzy Temporal Knowledge Representation and Reasoning. In Technologies for Constructing Intelligent Systems 2. Tools, Series Studies in Fuzziness and Soft Computing, pages 237-250, SpringerVerlag, 2002.

[48] I. Glöckner, Fuzzy Quantifiers - A Computational Theory, Springer, Berlin, 2006.

[49] F. Díaz-Hermida, A. Bugarín, P. Cariñena, and S. Barro, Voting-model based evaluation of fuzzy quantified sentences: a general framework, Fuzzy Sets and Systems, 146:97-120, Elsevier, 2004.

[50] L.A. Zadeh, The concept of a linguistic variable and its application to approximate reasoning. Parts 1-3, Information Sciences, 8:199-279, 301-357; 9:43-80, 1975. 